\title{
Position-momentum Einstein-Podolsky-Rosen state of distantly separated trapped atoms
}

\author{
A. S. Parkins \\ Department of Physics, University of Auckland, Private Bag 92019, Auckland, New Zealand \\ H. J. Kimble \\ Norman Bridge Laboratory of Physics 12-33, California Institute of Technology, Pasadena, California 91125
}

(Received 14 July 1999; published 5 April 2000)

\begin{abstract}
We propose a scheme for preparing an Einstein-Podolsky-Rosen state in position and momentum of a pair of distantly separated trapped atoms. The scheme utilizes the entangled light fields output from a nondegenerate optical parametric amplifier. Quantum-state exchange between these fields and the motional states of the trapped atoms is accomplished via interactions in cavity QED.
\end{abstract}

PACS number(s): 03.65.Bz, 03.67.Hk, 42.50.-p

\section{INTRODUCTION}

In 1935, Einstein, Podolsky, and Rosen (EPR) [1] proposed a now famous gedanken experiment involving a system of two particles spatially separated but correlated in position and momentum as described by a Wigner function of the form

$$
W\left(q_{1}, p_{1} ; q_{2}, p_{2}\right) \sim \delta\left(q_{1}+q_{2}\right) \delta\left(p_{1}-p_{2}\right),
$$

where $q_{1}$ and $q_{2}$ are the continuous position variables of the particles with corresponding conjugate momenta $p_{1}$ and $p_{2}$ [2]. That is, the positions and momenta of the two particles are perfectly anticorrelated $\left(q_{1}=-q_{2}\right)$ and correlated $\left(p_{1}\right.$ $\left.=p_{2}\right)$, respectively. With the assumption of local realism, but with the apparent ability to assign definite values to canonically conjugate variables of one particle from measurements of the other particle in this system, a conflict with the Heisenberg uncertainty principle seemingly follows, which led EPR to conclude that quantum mechanics is incomplete. Bohm [3] adapted this argument to a system of discrete (dichotomic) variables, to which Bell applied his classic analysis, deriving the so-called Bell inequalities [4-6] which quantify explicitly the conflict between local realism and quantum mechanics. Note that, although measurements of $\left(q_{i}, p_{i}\right)$ do not lead to a violation of a Bell inequality for the original EPR state of Eq. (1) (Ref. [5], p. 196), the entanglement of this state guarantees that an appropriate set of variables exists for which a contradiction with local realism would be manifest [7-9].

Experimental demonstrations of the conflict between quantum mechanics and local realism have concentrated almost exclusively on systems of discrete variables, such as electron spin or photon polarization $[6,10]$. Only one experiment, by Ou et al. [11,12], following suggestions by Reid and Drummond [13], has in fact realized the EPR paradox as originally envisioned by EPR; that is, for canonically conjugate variables with a continuous spectrum. The EPR "source" in this experiment was a nondegenerate optical parametric amplifier (NOPA), and the relevant variables were the quadrature amplitudes of the entangled electromagnetic fields generated in the parametric process. These am- plitudes are analogous to the position and momentum of a particle and can be measured very efficiently via homodyne detection [14].

In the present work, we describe a scheme that goes beyond an analogy and actually realizes an EPR state in position and momentum for a pair of massive particles at distinct physical locations. Our proposal for achieving stored entanglement for continuous quantum variables is based upon a set of interactions in cavity quantum electrodynamics (QED) that allows for the exchange of quantum states between the motion of trapped atoms and propagating light fields [15]. By exploiting these interactions and the light source of $[11,12]$, we show that it should be possible to prepare deterministically a state of the form (1) for a pair of trapped atoms located at macroscopically separated sites. Beyond conventional $(q, p)$ projections as in homodyne or heterodyne measurements, the setting of atom traps and cavity QED also enables detection strategies for the explicit demonstration of the nonlocal character of the resulting EPR state. Moreover, the techniques that we describe could be important resources for the realization of quantum networks, a particular example being the creation of EPR states to enable the teleportation of the center-of-mass wave function of a massive particle [16].

\section{TRAPPED ATOM COUPLED TO AN OPTICAL CAVITY MODE}

We begin with the basic setup that facilitates the motionlight coupling fundamental to our scheme [15]; this setup was originally considered by Zeng and Lin [17]. We consider a single two-level atom (or ion) confined in a harmonic trap located inside an optical cavity. The atomic transition of frequency $\omega_{a}$ is coupled to a single mode of the cavity field of frequency $\omega_{c}$ and is also assumed to be driven by an external (classical) laser field of frequency $\omega_{L}$. The physical setup and excitation scheme are depicted in Fig. 1. The cavity is aligned along the $x$ axis, while the laser field is incident from a direction in the $y-z$ plane (i.e., perpendicular to the $x$ axis).

The Hamiltonian describing the internal and external atomic degrees of freedom plus the atom-cavity and atomlaser couplings takes the form (in a frame rotating at the laser frequency for the internal-atomic and cavity operators) 

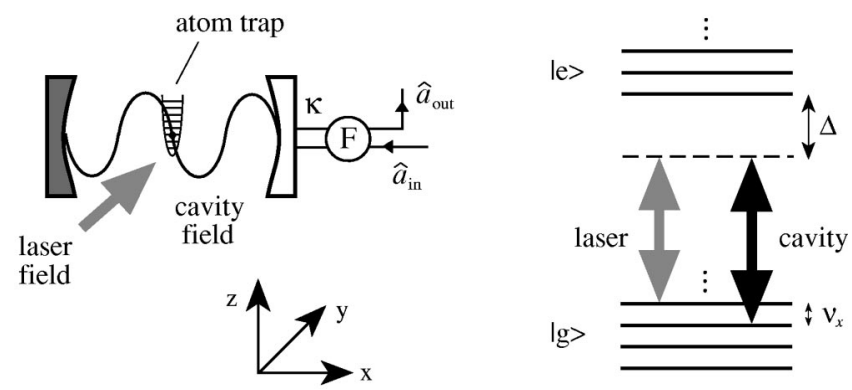

FIG. 1. Schematic of proposed experimental setup and excitation scheme for coupling between the motion of a trapped atom and a quantized cavity mode of the electromagnetic field, and thence to a freely propagating external field. Note that all input and output to the atom-cavity system is assumed to be through just one mirror; the other mirror is assumed to be perfect.

$$
\begin{aligned}
\hat{H}_{0}= & \sum_{j=x, y, z} \hbar \nu_{j}\left(\hat{b}_{j}^{\dagger} \hat{b}_{j}+1 / 2\right)+\hbar \delta \hat{a}^{\dagger} \hat{a}+\hbar \Delta \hat{\sigma}_{+} \hat{\sigma}_{-} \\
& +\hbar\left[\mathcal{E}_{L}(\hat{y}, \hat{z}, t) \hat{\sigma}_{+}+\mathcal{E}_{L}^{*}(\hat{y}, \hat{z}, t) \hat{\sigma}_{-}\right] \\
& +\hbar g_{0} \sin (k \hat{x})\left(\hat{a}^{\dagger} \hat{\sigma}_{-}+\hat{\sigma}_{+} \hat{a}\right) .
\end{aligned}
$$

Here, $\left\{\nu_{x}, \nu_{y}, \nu_{z}\right\}$ are the harmonic oscillation frequencies along the principal axes of the trap, $\hat{b}_{j}$ and $\hat{a}$ are annihilation operators for the quantized atomic motion and cavity field, respectively, $\hat{\sigma}_{-}=|g\rangle\langle e|$ is the atomic lowering operator, and $\delta=\omega_{c}-\omega_{L}$ and $\Delta=\omega_{a}-\omega_{L}$. The quantity $\mathcal{E}_{L}(\hat{y}, \hat{z}, t)$ is the (possibly time-dependent) amplitude of the laser field. The single-photon atom-cavity dipole coupling strength is given by $g_{0}$, while the sine function describes the standing wave structure of the cavity field (we assume that the center of the trap is located at a node of the cavity field), with $k$ $=2 \pi / \lambda$ the wave number of the field and $\hat{x}$ $=\left[\hbar /\left(2 m \nu_{x}\right)\right]^{1 / 2}\left(\hat{b}_{x}+\hat{b}_{x}^{\dagger}\right)$.

In [15] a number of assumptions and approximations are made in order to simplify the model. In particular, (1) The detunings of the light fields from the atomic transition frequency are assumed to be very large (i.e., $\Delta \gg\left|\mathcal{E}_{L}\right|, g_{0}, \delta, \nu_{j}$ ), enabling atomic spontaneous emission to be neglected and the internal atomic dynamics to be adiabatically eliminated. (2) Any forms of motional decoherence associated with the trap itself are ignored. (3) The size of the harmonic trap is assumed to be small compared to the optical wavelength (Lamb-Dicke regime), enabling the approximations $\sin (k \hat{x})$ $\simeq \eta_{x}\left(\hat{b}_{x}+\hat{b}_{x}^{\dagger}\right)$, where $\eta_{x}(\ll 1)$ is the Lamb-Dicke parameter, and $\mathcal{E}_{L}(\hat{y}, \hat{z}, t) \simeq \mathcal{E}_{L}(t) e^{-i \phi_{L}}$. (4) The cavity and laser fields are tuned so that $\delta=\omega_{c}-\omega_{L}=\nu_{x}$. (5) The trap frequency $\nu_{x}$ and cavity field decay rate $\kappa_{a}$ are assumed to satisfy $\nu_{x}$ $\gg \kappa_{a} \gg\left|\left(g_{0} \eta_{x} / \Delta\right) \mathcal{E}_{L}(t)\right|$. The first inequality allows a rotating-wave approximation to be made with respect to the trap oscillation frequency, while the second inequality enables an adiabatic elimination of the cavity field mode.

Given these conditions one can show that the motional mode dynamics in the $x$ direction can be described by the simple quantum Langevin equation

$$
\dot{\vec{b}}_{x} \simeq-\Gamma(t) \widetilde{b}_{x}+\sqrt{2 \Gamma(t)} \widetilde{a}_{\mathrm{in}}(t),
$$

where $\widetilde{b}_{x}=e^{i \nu_{x} t} \hat{b}_{x}, \Gamma(t)=\left[g_{0} \eta_{x} \mathcal{E}_{L}(t) / \Delta\right]^{2} / \kappa_{a}$, and $\tilde{a}_{\text {in }}(t)$, which satisfies the commutation relation $\left[\widetilde{a}_{\text {in }}(t), \widetilde{a}_{\text {in }}^{\dagger}\left(t^{\prime}\right)\right]$ $=\delta\left(t-t^{\prime}\right)$, is the quantum noise operator describing the input to the cavity field (in a frame rotating at the cavity frequency). In this way, the statistics of the input light field can be "written onto" the state of the oscillator. In [15] it was shown how this effect can be used to efficiently prepare a squeezed state of the motion of the trapped atom. Here we extend that work further to the generation of entanglement between the motional states of trapped atoms at separated sites. In particular, our protocol transfers entanglement from a pair of quantum-correlated light fields to a pair of trapped atoms in a process of quantum state exchange, or qusex.

\section{LIGHT SOURCE: NONDEGENERATE PARAMETRIC AMPLIFIER}

Our source of quantum-correlated light fields is taken to be a NOPA operating below threshold $[11,12,14]$. The light fields may be nondegenerate in polarization or in frequency. We denote the annihilation operators for the two intracavity field modes, of frequencies $\omega_{1}$ and $\omega_{2}$, by $\hat{c}_{1}$ and $\hat{c}_{2}$, and the Hamiltonian describing the coupling between these modes takes the form (in a rotating frame)

$$
H_{\mathrm{I}}=i \hbar \epsilon\left(\hat{c}_{1} \hat{c}_{2}-\hat{c}_{1}^{\dagger} \hat{c}_{2}^{\dagger}\right)
$$

where $\epsilon$ is the coupling strength, proportional to the nonlinear susceptibility of the intracavity medium and to the strength of the coherent pump field (at frequency $\omega_{1}+\omega_{2}$ ).

Assuming the cavity mode amplitudes to be damped at the same rate $\kappa_{c}$, equations of motion for the mode operators (in the rotating frame) can be derived as

$$
\dot{\hat{c}}_{1,2}=-\kappa_{c} \hat{c}_{1,2}-\epsilon \hat{c}_{2,1}^{\dagger}+\sqrt{2 \kappa_{c}} \hat{c}_{\text {in }}^{(1,2)}(t),
$$

where $\hat{c}_{\text {in }}^{(1,2)}(t)$ are the vacuum input fields to the NOPA cavity modes (see, e.g., $[18,19]$ ). The output fields from the NOPA then follow from the boundary conditions

$$
\hat{c}_{\text {out }}^{(1,2)}(t)+\hat{c}_{\text {in }}^{(1,2)}(t)=\sqrt{2 \kappa_{c}} \hat{c}_{1,2}(t) .
$$

The (linear) equations above are readily solved in a Fouriertransformed space defined by $Z(\omega)$ $=(2 \pi)^{-1 / 2} \int d t Z(t) e^{-i \omega t}$. Defining quadrature phase amplitudes ("positions" and "momenta') for the output fields by

$$
\begin{gathered}
X_{\mathrm{out}}^{(1,2)}(t)=\hat{c}_{\mathrm{out}}^{(1,2)}(t)+\hat{c}_{\mathrm{out}}^{(1,2) \dagger}(t), \\
Y_{\mathrm{out}}^{(1,2)}(t)=-i\left[\hat{c}_{\mathrm{out}}^{(1,2)}(t)-\hat{c}_{\mathrm{out}}^{(1,2) \dagger}(t)\right],
\end{gathered}
$$

the sum of the $X$ amplitudes is derived as [14]

$$
\begin{aligned}
X_{\mathrm{out}}^{(1)}(\omega)+X_{\mathrm{out}}^{(2)}(\omega) & =\frac{\kappa_{c}-\epsilon+i \omega}{\kappa_{c}+\epsilon-i \omega}\left[X_{\mathrm{in}}^{(1)}(\omega)+X_{\mathrm{in}}^{(2)}(\omega)\right] \\
& \rightarrow 0 \quad \text { as } \epsilon \rightarrow \kappa_{c} \quad \text { and } \quad \omega \rightarrow 0,
\end{aligned}
$$




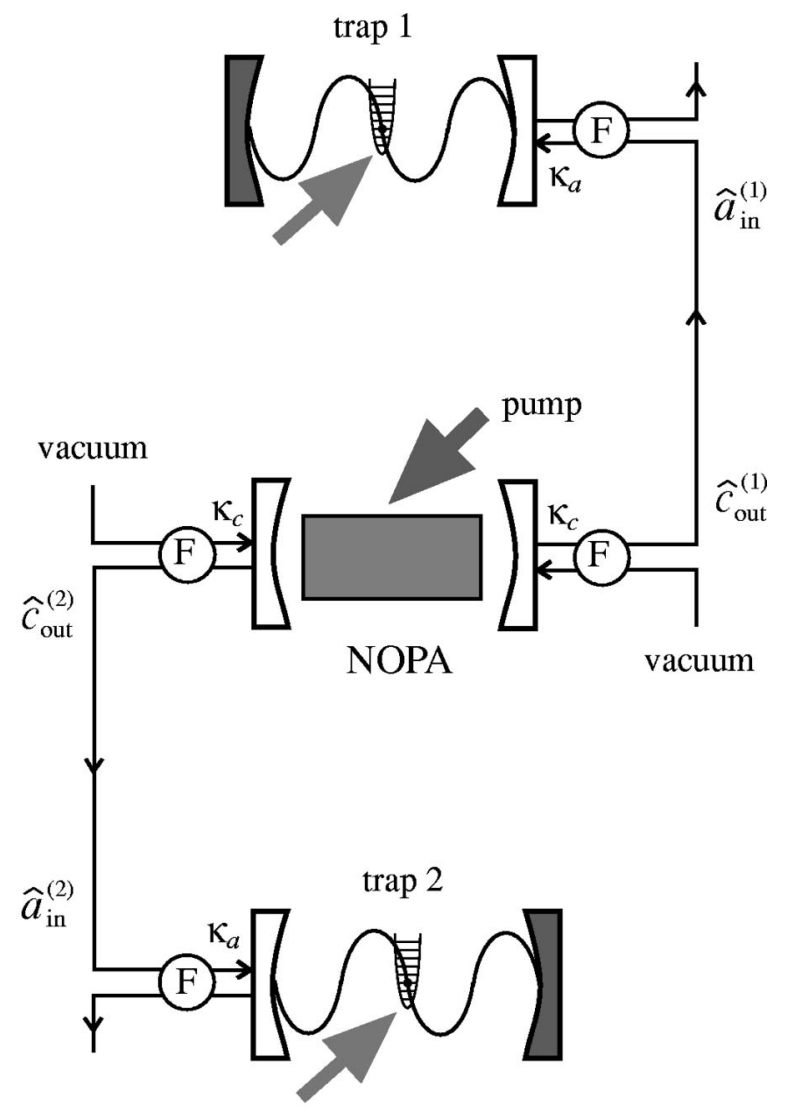

FIG. 2. Preparation of an EPR state of the motion of two separated trapped atoms. The output modes from a nondegenerate parametric amplifier (NOPA) are incident on two separated trappedatom-cavity configurations of the type depicted in Fig. 1. Faraday isolators $(F)$ facilitate a unidirectional coupling between the entangled light source and the atom-cavity systems. Note that in practice the outputs from the two NOPA modes might actually be through the same mirror, but could be separated due to differing polarizations or frequencies.

while the difference of the $Y$ amplitudes is

$$
\begin{aligned}
Y_{\mathrm{out}}^{(1)}(\omega)-Y_{\mathrm{out}}^{(2)}(\omega) & =\frac{\kappa_{c}-\epsilon+i \omega}{\kappa_{c}+\epsilon-i \omega}\left[Y_{\mathrm{in}}^{(1)}(\omega)-Y_{\mathrm{in}}^{(2)}(\omega)\right] \\
& \rightarrow 0 \quad \text { as } \epsilon \rightarrow \kappa_{c} \quad \text { and } \quad \omega \rightarrow 0 .
\end{aligned}
$$

So the two output fields are highly correlated and, close to $\omega=0$ and for $\epsilon \rightarrow \kappa_{c}$, their quadrature amplitudes exhibit precisely the properties of the original EPR state, as demonstrated explicitly by the Wigner function for the state of the fields $[12,19]$.

\section{LIGHT-TO-MOTION QUANTUM-STATE EXCHANGE}

As depicted in Fig. 2, the two NOPA output fields are assumed to be incident on separate cavities, each containing a trapped atom in the configuration described earlier. Note that the output fields from the NOPA are resonant with the respective cavity mode frequencies. We assume that $\Gamma(t)$ $=\Gamma$, a constant, and, for simplicity, that $\Gamma$ is the same for both configurations. Denoting the motional mode operators for the two atoms along the $x$ axis by $\widetilde{b}_{1 x}$ and $\widetilde{b}_{2 x}$, respectively, the two systems are thus described by $[20,21]$

$$
\begin{gathered}
\dot{\vec{b}}_{j x}=-\Gamma \widetilde{b}_{j x}+\sqrt{2 \Gamma} \widetilde{a}_{\text {in }}^{(j)}(t)=-\Gamma \widetilde{b}_{j x}+\sqrt{2 \Gamma} \widetilde{c}_{\text {out }}^{(j)}(t-\tau) \\
(j=1,2),
\end{gathered}
$$

where $\tau$ is a time delay (assumed the same for both cavities); provided the coupling between the NOPA and the cavities is unidirectional, this delay can essentially be ignored [20,21].

If the bandwidths of the input light fields from the NOPA are sufficiently broad, in particular if $\kappa_{c} \gg \Gamma$ [i.e., $\widetilde{c}_{\text {out }}^{(1,2)}(t)$ can be regarded as quantum white noise operators in Eq. (11)], then one can perform an average over the input fields and derive a master equation (see, e.g., $[18,19])$ for the density operator $\rho$ of the motional modes alone,

$$
\begin{aligned}
\dot{\rho}= & \Gamma(N+1)\left(2 \widetilde{b}_{1 x} \rho \widetilde{b}_{1 x}^{\dagger}-\widetilde{b}_{1 x}^{\dagger} \widetilde{b}_{1 x} \rho-\rho \widetilde{b}_{1 x}^{\dagger} \widetilde{b}_{1 x}\right) \\
& +\Gamma N\left(2 \widetilde{b}_{1 x}^{\dagger} \rho \widetilde{b}_{1 x}-\widetilde{b}_{1 x} \widetilde{b}_{1 x}^{\dagger} \rho-\rho \widetilde{b}_{1 x} \widetilde{b}_{1 x}^{\dagger}\right) \\
& +\Gamma(N+1)\left(2 \widetilde{b}_{2 x} \rho \widetilde{b}_{2 x}^{\dagger}-\widetilde{b}_{2 x}^{\dagger} \widetilde{b}_{2 x} \rho-\rho \widetilde{b}_{2 x}^{\dagger} \widetilde{b}_{2 x}\right) \\
& +\Gamma N\left(2 \widetilde{b}_{2 x}^{\dagger} \rho \widetilde{b}_{2 x}-\widetilde{b}_{2 x} \widetilde{b}_{2 x}^{\dagger} \rho-\rho \widetilde{b}_{2 x} \widetilde{b}_{2 x}^{\dagger}\right) \\
& +2 \Gamma M\left(\widetilde{b}_{1 x} \rho \widetilde{b}_{2 x}+\widetilde{b}_{2 x} \rho \widetilde{b}_{1 x}-\widetilde{b}_{1 x} \widetilde{b}_{2 x} \rho-\rho \widetilde{b}_{1 x} \widetilde{b}_{2 x}\right) \\
& +2 \Gamma M\left(\widetilde{b}_{1 x}^{\dagger} \rho \widetilde{b}_{2 x}^{\dagger}+\widetilde{b}_{2 x}^{\dagger} \rho \widetilde{b}_{1 x}^{\dagger}-\widetilde{b}_{1 x}^{\dagger} \widetilde{b}_{2 x}^{\dagger} \rho-\rho \widetilde{b}_{1 x}^{\dagger} \widetilde{b}_{2 x}^{\dagger}\right),
\end{aligned}
$$

with the parameters $N$ and $M$ given, in terms of the NOPA parameters, by

$$
N=\frac{4 \epsilon^{2} \kappa_{c}^{2}}{\left(\kappa_{c}^{2}-\epsilon^{2}\right)^{2}}, \quad M=2 \kappa_{c} \epsilon \frac{\kappa_{c}^{2}+\epsilon^{2}}{\left(\kappa_{c}^{2}-\epsilon^{2}\right)^{2}} .
$$

This master equation has a steady-state solution

$$
\rho^{s s}=\left|\psi_{12}\right\rangle\left\langle\psi_{12}\right|
$$

i.e., a pure state, with

$$
\begin{aligned}
\left|\psi_{12}\right\rangle & =S_{12}(r)|0\rangle_{1 x}|0\rangle_{2 x} \\
& =[\cosh (r)]^{-1} \sum_{m=0}^{\infty}[-\tanh (r)]^{m}|m\rangle_{1 x}|m\rangle_{2 x},
\end{aligned}
$$

where $|m\rangle_{1 x, 2 x}$ are Fock states of the motional modes and $S_{12}(r)$ is the two-mode squeezing operator [19],

$$
S_{12}(r)=\exp \left[r\left(\widetilde{b}_{1 x} \widetilde{b}_{2 x}-\widetilde{b}_{1 x}^{\dagger} \widetilde{b}_{2 x}^{\dagger}\right)\right] .
$$

This operator transforms the mode annihilation operators for the atomic motion as

$$
\begin{aligned}
& S_{12}^{\dagger}(r) \widetilde{b}_{1 x} S_{12}(r)=\cosh (r) \widetilde{b}_{1 x}-\sinh (r) \widetilde{b}_{2 x}^{\dagger}, \\
& S_{12}^{\dagger}(r) \widetilde{b}_{2 x} S_{12}(r)=\cosh (r) \widetilde{b}_{2 x}-\sinh (r) \widetilde{b}_{1 x}^{\dagger},
\end{aligned}
$$


where $\cosh (r)=\sqrt{N+1}$ and $\sinh (r)=\sqrt{N}$. Defining position and momentum operators as

$$
Q_{j}=\widetilde{b}_{j x}+\tilde{b}_{j x}^{\dagger}, P_{j}=-i\left(\tilde{b}_{j x}-\widetilde{b}_{j x}^{\dagger}\right),
$$

it follows that

$$
\begin{gathered}
S_{12}^{\dagger}(r)\left(Q_{1}+Q_{2}\right) S_{12}(r)=e^{-r}\left(Q_{1}+Q_{2}\right), \\
S_{12}^{\dagger}(r)\left(P_{1}-P_{2}\right) S_{12}(r)=e^{-r}\left(P_{1}-P_{2}\right),
\end{gathered}
$$

and so, in the limit $\epsilon \rightarrow \kappa_{c}$ (i.e., $r \rightarrow \infty$ ), an EPR state in the positions and momenta of the two trapped atoms is established.

The nature of the correlations inherent in the joint state (15) of the atomic motion is most clearly expressed through the Wigner function for this state $[12,19]$ :

$$
\begin{aligned}
W\left(q_{1}, p_{1} ; q_{2}, p_{2}\right)= & \frac{4}{\pi^{2}} \exp \left\{-\left[\left(q_{1}+q_{2}\right)^{2}+\left(p_{1}-p_{2}\right)^{2}\right] e^{+2 r}\right\} \\
& \times \exp \left\{-\left[\left(q_{1}-q_{2}\right)^{2}+\left(p_{1}+p_{2}\right)^{2}\right] e^{-2 r}\right\} \\
\sim & C \delta\left(q_{1}+q_{2}\right) \delta\left(p_{1}-p_{2}\right) \quad \text { as } \quad r \rightarrow \infty,
\end{aligned}
$$

with $C$ a constant. This entangled state is achieved in steady state over a time $t \gg \Gamma^{-1}$. The coupling to the external fields from the NOPA can then be turned off by setting $\mathcal{E}_{L 1,2}$ to zero. The result is a stored EPR state for the motion of two trapped atoms that would persist for a duration set by the time scale for motional decoherence.

\section{DISCUSSION}

Before considering some of the interesting possibilities offered by this system, we return briefly to some of the major assumptions associated with the model. First, the finite effect of atomic spontaneous emission events on the motional state can be estimated as in [15]. This effect can be neglected provided the rate of these events is much smaller than the rate $\Gamma$ at which the motional steady state is achieved. The condition one derives by enforcing this inequality amounts to the condition of a "one-dimensional" atom in cavity QED, $C_{1}=g_{0}^{2} /\left(\kappa_{a} \gamma\right) \gg 1$, where $\gamma$ is the linewidth (full width at half maximum) of the atomic transition [22]. Note that the recent experiments of Refs. [23-25] have achieved $C_{1}$ $\simeq 70,28$, and 50 , respectively. With regards to the trapping potential, harmonic frequencies on the order of tens of $\mathrm{MHz}$ have been achieved in ion traps, with corresponding LambDicke parameters on the order of 0.1 and smaller [27]. Note, however, that large values of the entanglement parameter $r$ imply population of large- $m$ number states and a broader spread of the atomic wave packet. Given that the mean excitation number for the state (15) is $N=\sinh ^{2}(r)$, a more precise form of the Lamb-Dicke assumption would be $\eta_{x} \sqrt{N+1}=\eta_{x} \cosh (r) \ll 1$.

With trap frequencies such as those quoted above, the condition $v_{x} \gg \kappa_{a}$ should be satisfied for a cavity field decay rate of a few $\mathrm{MHz}$ or less; such values of $\kappa_{a}$ have been approached in the experiments of Refs. [24], [25], and potentially could be realized with improved cavity finesse as in Ref. [26]. Assuming that this is the case, likely magnitudes for the rate $\Gamma$ would then be tens or hundreds of $\mathrm{kHz}$. Finally, time scales for motional decoherence and heating in recent ion trap experiments are of the order of milliseconds, with further improvement likely [27]; given the various rates discussed above, these effects would not be expected to hamper the preparation of the entangled state.

As for applications of this system, further investigation of the EPR paradox would obviously be possible, with a variety of motional state measurements able to be implemented with high efficiency on the trapped atoms [28,29], possibly also via the cavity field [15]. In particular, violations of a Bell inequality for the state (15) can be obtained with measurements that project onto a basis of even and odd phonon number (i.e., that measure the parity operator) [7-9], or that simply distinguish between states with no phonons $(|m=0\rangle)$ and one or more phonons $(|m>0\rangle)$ [9], for each of the trapped atoms [30]. To the extent that a "macroscopic" number of quanta may in principle be involved, such investigation could also address new viewpoints on the compatibility of quantum mechanics with local realism [31].

On a somewhat more applied side is the possibility of using the EPR state (15) for quantum dense coding [32], or for the teleportation of the quantum state of a system with continuous variables [33,34], generalizing the original discrete-variable teleportation protocol of Bennett et al. [35]. This elegant adaptation of the EPR paradox has in fact been realized with light fields, again using optical parametric amplifiers and homodyne measurements of quadrature amplitudes [36]. The scheme outlined in this paper opens the door to teleporting an atomic center-of-mass wave function [16], by providing the motional state entanglement required by the continuous-variable teleportation protocol.

Such a capability is also of considerable interest in the related context of quantum computation with trapped atoms and light $[37,38]$. Here, we specifically have in mind protocols that combine quantum information processing with both discrete and continuous variables [39]. Any implementation of a qubit (e.g., internal atomic states or photon polarization) could be linked with an external degree of freedom (e.g., atomic center-of-mass or complex amplitude of the electromagnetic field), with the complete system viewed as a composite unit (qubit plus qunat [39]) for protocols such as quantum communication between distant nodes of a quantum network [15,40-43].

Further to this theme, note also that we need not restrict ourselves to a single trapped atom at each site. For example, if there are $K$ atoms inside each cavity, then focusing the coupling lasers $\left(\mathcal{E}_{L 1,2}\right)$ sequentially on atoms $1,2, \ldots, K$ at each site (and neglecting any direct interaction between neighboring atoms at each site) would generate a set of pairwise EPR-entangled atoms. Alternatively, and perhaps more interestingly, one might consider the case in which the $K$ atoms at each site are simultaneously coupled to the cavity. 
Assuming for simplicity that they have identical coupling strengths, then the system would again be described by Eq. (12), but with the replacements $\widetilde{b}_{1,2 x} \rightarrow \widetilde{B}_{1,2 x}$ $\equiv K^{-1 / 2} \sum_{j=1}^{K} \widetilde{b}_{1,2 x}^{(j)}$ and $\Gamma \rightarrow K \Gamma$. Such dynamics evidently leads to a highly entangled state of all $2 K$ atoms, a situation of potentially great utility and, indeed, of considerable general interest.

\section{ACKNOWLEDGMENTS}

A. S. P. gratefully acknowledges support from the Marsden Fund of the Royal Society of New Zealand. H. J. K. is supported by the National Science Foundation, by DARPA via the QUIC Institute which is administered by ARO, and by the Office of Naval Research.
[1] A. Einstein, B. Podolsky, and N. Rosen, Phys. Rev. 47, 777 (1935).

[2] The $\delta$-function form of the Wigner function corresponding to the original EPR state is physically idealized and somewhat problematic from a mathematical point of view, since it cannot be normalized. The symbol $\delta$ should thus be regarded as representing a normalizable function with an arbitrarily high and narrow peak. See, for example, O. Cohen, Phys. Rev. A 56, 3484 (1997).

[3] D. Bohm, Quantum Theory (Prentice-Hall, Englewood Cliffs, NJ, 1951).

[4] J. S. Bell, Physics (Long Island City, N.Y.) 1, 195 (1965).

[5] J. S. Bell, Speakable and Unspeakable in Quantum Mechanics (Cambridge University Press, London, 1987).

[6] J. F. Clauser and A. Shimony, Rep. Prog. Phys. 41, 1881 (1978).

[7] A. Peres, Quantum Theory: Concepts and Methods (Kluwer, Dordrecht, 1993).

[8] K. Banaszek and K. Wódkiewicz, Phys. Rev. A 58, 4345 (1998).

[9] K. Banaszek and K. Wódkiewicz, Acta Phys. Slov. 49, 491 (1999).

[10] See, for example, A. Aspect, J. Dalibard, and G. Roger, Phys. Rev. Lett. 49, 1804 (1982); Z. Y. Ou and L. Mandel, ibid. 61, 50 (1988); Y. H. Shih and C. O. Alley, ibid. 61, 2921 (1988); J. G. Rarity and P. R. Tapster, ibid. 64, 2495 (1990).

[11] Z. Y. Ou, S. F. Pereira, H. J. Kimble, and K. C. Peng, Phys. Rev. Lett. 68, 3663 (1992).

[12] Z. Y. Ou, S. F. Pereira, and H. J. Kimble, Appl. Phys. B: Photophys. Laser Chem. 55, 265 (1992).

[13] M. D. Reid and P. D. Drummond, Phys. Rev. Lett. 60, 2731 (1988); M. D. Reid, Phys. Rev. A 40, 913 (1989); P. D. Drummond and M. D. Reid, ibid. 41, 3930 (1990).

[14] H. J. Kimble, in Fundamental Systems in Quantum Optics, Proceedings of the Les Houches Summer School of Theoretical Physics, Session LIII, Les Houches, 1990, edited by J. Dalibard et al. (Elsevier, New York, 1992).

[15] A. S. Parkins and H. J. Kimble, J. Opt. B: Quantum Semiclass. Opt. 1, 496 (1999).

[16] A. S. Parkins and H. J. Kimble, e-print quant-ph/9909021.

[17] H. Zeng and F. Lin, Phys. Rev. A 50, R3589 (1994).

[18] C. W. Gardiner, Quantum Noise (Springer, Berlin, 1991).

[19] D. F. Walls and G. J. Milburn, Quantum Optics (SpringerVerlag, Berlin, 1994).

[20] C. W. Gardiner, Phys. Rev. Lett. 70, 2269 (1993).

[21] H. J. Carmichael, Phys. Rev. Lett. 70, 2273 (1993).

[22] Q. A. Turchette et al., Phys. Rev. Lett. 75, 4710 (1995).
[23] C. J. Hood, M. S. Chapman, T. W. Lynn, and H. J. Kimble, Phys. Rev. Lett. 80, 4157 (1998).

[24] P. Münstermann et al., Phys. Rev. Lett. 82, 3791 (1999).

[25] J. Ye, D. W. Vernooy, and H. J. Kimble, Phys. Rev. Lett. 83, 4987 (1999).

[26] G. Rempe, R. J. Thompson, H. J. Kimble, and R. Lalezari, Opt. Lett. 17, 363 (1992).

[27] D. J. Wineland et al., J. Res. Natl. Inst. Stand. Technol. 103, 259 (1998).

[28] D. Leibfried et al., Phys. Rev. Lett. 77, 4281 (1996), and references therein.

[29] S. A. Gardiner, J. I. Cirac, and P. Zoller, Phys. Rev. A 55, 1683 (1997).

[30] To be more precise, these measurements follow small (local) coherent displacements of the trapped atoms. Since the measurements are of dichotomic observables, one can construct a Bell-type inequality similar to that for two entangled spin-1/2 particles, and the coherent displacement amplitudes enter as the adjustable parameters (cf. polarizer orientations for spin$1 / 2$ particles).

[31] M. D. Reid, Europhys. Lett. 36, 1 (1996).

[32] C. H. Bennett and S. J. Wiesner, Phys. Rev. Lett. 69, 2881 (1992).

[33] L. Vaidman, Phys. Rev. A 49, 1473 (1994); see also L. Vaidman and N. Yoran, ibid. 59, 116 (1999).

[34] S. L. Braunstein and H. J. Kimble, Phys. Rev. Lett. 80, 869 (1998).

[35] C. H. Bennett et al., Phys. Rev. Lett. 70, 1895 (1993).

[36] A. Furusawa et al., Science 282, 706 (1998).

[37] For quantum computing using internal atomic states and external vibrational states, see, e.g., J. I. Cirac and P. Zoller, Phys. Rev. Lett. 74, 4091 (1995); C. Monroe et al., ibid. 75, 4714 (1995); Q. A. Turchette et al., ibid. 81, 3631 (1998); and Ref. [27].

[38] For quantum computing using internal atomic states and cavity QED, see, e.g., T. Pellizzari, S. A. Gardiner, J. I. Cirac, and P. Zoller, Phys. Rev. Lett. 75, 3788 (1995); S. J. van Enk, J. I. Cirac, and P. Zoller, ibid. 79, 5178 (1997).

[39] Conditions for the construction of a universal quantum computer over continuous variables have been given by S. Lloyd and S. L. Braunstein, Phys. Rev. Lett. 82, 1784 (1999).

[40] J. I. Cirac, P. Zoller, H. J. Kimble, and H. Mabuchi, Phys. Rev. Lett. 78, 3221 (1997).

[41] S. J. van Enk, J. I. Cirac, and P. Zoller, Phys. Rev. Lett. 78, 4293 (1997); Science 279, 205 (1998).

[42] T. Pellizzari, Phys. Rev. Lett. 79, 5242 (1997).

[43] S. J. van Enk, H. J. Kimble, J. I. Cirac, and P. Zoller, Phys. Rev. A 59, 2659 (1999). 